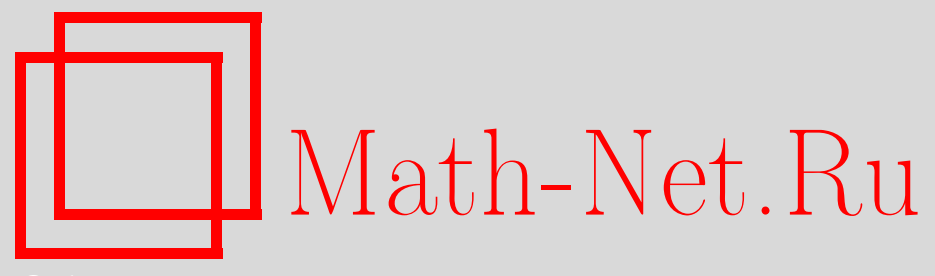

C. А. Бочкарев, С. В. Лекомцев, Аэроупругая устойчивость пластины, взаимодействующей с текущей жидкостью, Вестн. Сам. гос. техн. ун-та. Сер. Физ.-мат. науки, 2016, номер 3, 552-566

DOI: https://doi.org/10.14498/vsgtu1510

Использование Общероссийского математического портала MathNet.Ru подразумевает, что вы прочитали и согласны с пользовательским соглашением

http://www.mathnet.ru/rus/agreement

Параметры загрузки:

IP: 52.6 .47 .48

26 апреля 2023 г., 18:15:53

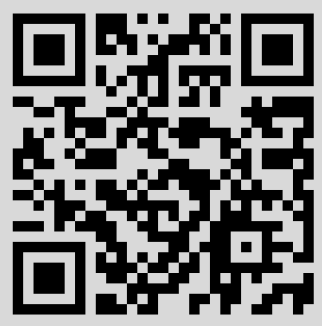


УДК 533.6.013.42

\title{
АЭРОУПРУГАЯ УСТОЙЧИВОСТЬ ПЛАСТИНЫ, ВЗАИМОДЕЙСТВУЮЩЕЙ С ТЕКУЩЕЙ ЖИДКОСТЬЮ
}

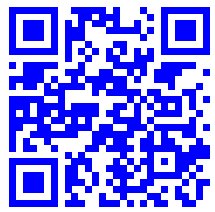

\author{
С. А. Бочкарев, С. В. Лекомиев
}

Институт механики сплошных сред УрО РАН, Россия, 614013, Пермь, ул. Акад. Королёва, 1.

\begin{abstract}
Аннотация
Представлены результаты численного исследования динамического поведения деформируемой пластины, которая взаимодействует одновременно с внешним сверхзвуковым потоком газа и внутренним потоком жидкости. Основные уравнения, описывающие поведение идеальной сжимаемой жидкости в случае малых возмущений, записываются в терминах потенциала возмущенных скоростей и преобразуются с использованием метода Бубнова-Галеркина. Аэро- и гидродинамическое давления вычисляются согласно квазистатической аэродинамической теории и формуле Бернулли. Деформации пластины определяются с помощью теории, основанной на гипотезах Тимошенко. Математическая постановка задачи динамики упругой конструкции выполнена с использованием вариационного принципа возможных перемещений, в который включаются выражения для работы аэро- и гидродинамических сил. Вычисление комплексных собственных значений связанной системы двух уравнений осуществляется с помощью алгоритма на основе неявно перезапускаемого метода Арнольди. Оценка устойчивости основана на анализе комплексных собственных значений системы уравнений, полученной при последовательно возрастающей скорости течения жидкости или газа. Достоверность решения задачи подтверждена сравнением с известными численными и аналитическими результатами. Продемонстрировано существование различных видов неустойчивости в зависимости от скорости течения обоих потоков, задаваемых на краях пластины комбинаций кинематических граничных условий и высоты слоя жидкости. Установлено, что нарушение гладкости полученных зависимостей и диаграмм устойчивости обусловлено либо сменой моды флаттера, либо сменой типа потери устойчивости.
\end{abstract}

Ключевые слова: аэроупругость, сверхзвуковой поток газа, потенциальная сжимаемая жидкость, прямоугольная пластина, метод конечных элементов, устойчивость, флаттер.

(C) 2016 Самарский государственный технический университет.

\section{Образец для цитирования}

Боч к ар ев С. А., Л ек о м е в С. В. Аэроупругая устойчивость пластины, взаимодействующей с текущей жидкостью // Вестн. Сам. гос. техн. ун-та. Сер. Физ.-мат. науки, 2016. T. 20, № 3. C. 552-566. doi: 10.14498/vsgtu1510.

\section{Сведения об авторах}

Бочкарев Сергей Аркадъевич (к.ф.-м.н.; bochkarev@icmm.ru; автор, ведущие переписку), старший научный сотрудник, отдел комплексных проблем механики деформируемых твердых тел.

Лекомиев Сергей Владимирович (к.ф.-м.н.; lekomtsev@icmm.ru), научный сотрудник, отдел комплексных проблем механики деформируемых твердых тел. 
Введение. Интенсивные теоретические и экспериментальные исследования [1-6], проведенные за последние десятилетия, позволили получить обширные сведения о динамических процессах, протекающих в тонкостенных пластинах, обтекаемых потоком газа. Достигнутые успехи в изучении явления потери устойчивости конструкций, возникающей при превышении скоростью течения пороговой величины, в немалой степени обязаны построенному на основе поршневой теории аналитическому выражению для аэродинамического давления $[1,2]$. Сегодня такой подход, по сути дела, является основным инструментом, применяемым многими авторами для изучения панельного флаттера в случае сверхзвукового течения, в том числе и при использовании конечно-элементных алгоритмов [7]. При дозвуковом течении газа его поведение описывается в рамках потенциальной теории. Полученные результаты показали, что тип неустойчивости определяется не только параметрами течения, но и кинематическими граничными условиями, задаваемыми на краях пластины. Было установлено [8,9], что свободно опертая и жестко защемленная пластины теряют устойчивость в виде дивергенции в дозвуковом потоке газа и в виде флаттера в сверхзвуковом. В случае консольного закрепления неустойчивость наступает в виде флаттера. История изучения этого явления достаточно подробно отражена в монографии [8].

Учет нелинейных членов в соотношениях для деформаций позволил авторам более точно моделировать колебания большой амплитуды, возникающие В процессе флаттера [10-14]. В [12,13] нелинейная проблема на собственные значения сводится к последовательности линейных задач с помощью итерационного алгоритма, основанного на построении собственных форм колебаний. Аэроупругая устойчивость пластин, выполненных из функциональноградиентных (ФГ) и композиционных материалов, рассмотрена в [14-16]. В работах $[14,15]$ оценено влияние показателя объемной доли на величину критического динамического давления газа. В ходе решения нелинейной задачи в [14] обнаружено, что ФГ материал существенно изменяет поведение конструкции в закритической зоне. В [15] показано, что температурный нагрев приводит к снижению критических скоростей флаттера. Анализ анизотропных пластин выполнен в [16]. Здесь показано, что немонотонная зависимость собственных частот колебаний от угла намотки волокна приводит к аналогичному качественному изменению критических значений аэродинамического давления.

Исследования пластин, взаимодействующих с текущей жидкостью, также имеют более чем полувековую историю. Изучению этого феномена с помощью численно-аналитических методов посвящено значительное количество работ, начиная от анализа поведения бесконечных или полубесконечных пластин и заканчивая анализом конечномерных пространственных конструкций. История развития методов решения и моделей отражена в статьях $[17,18]$ и монографии [8]. Трехмерная постановка задачи о гидроупругой устойчивости пластин и ее решение методом конечных элементов (МКЭ) выполнены в работах [17-19]. Здесь проанализировано влияние на критические скорости потери устойчивости нескольких вариантов кинематических граничных условий, задаваемых на краях одиночной $[17,19]$ или входящей в сборку пластины [18, 19]. В работе [17] дополнительно рассмотрена расчетная схема, где 
жидкость в направлении потока не ограничена по размерам пластины. Полученные результаты показали, что для представленных комбинаций граничных условий используемое допущение об ограниченности объема жидкости приводит к незначительно заниженным значениям критических скоростей дивергенции и завышенным скоростям флаттера.

Приведенные в литературе исследования аэроупругой устойчивости пластин, взаимодействующих с жидкостью, весьма ограничены. Комбинация метода конечных и граничных элементов используется в [20] для моделирования динамического взаимодействия упругой пластины со сверхзвуковым потоком газа снизу и со слоем неподвижной жидкости сверху. Аэродинамическое давление вычисляется согласно поршневой теории. Поведение жидкости с учетом плескания свободной поверхности описывается уравнениями потенциальной теории, для численного решения которых применяется метод граничных элементов. Результаты продемонстрировали, что критические скорости газа и мода, по которой осуществляется потеря устойчивости, зависят от высоты слоя жидкости.

При анализе устойчивости цилиндрических оболочек, взаимодействующих одновременно с внешним сверхзвуковым потоком газа и внутренним потоком жидкости, было установлено [21], что комбинированное воздействие не только влияет на критические скорости, но и приводит к смене типа потери устойчивости. Можно предположить, что аналогичные эффекты будут наблюдаться и для пластины. Таким образом, целью данной работы является как проверка этой гипотезы, так и анализ других факторов, оказывающих влияние на границы устойчивости.

1. Постановка задачи. Рассматривается прямоугольная упругая пластина толщиной $h$, которая обтекается сверху сверхзвуковым потоком газа, а снизу взаимодействует с текущей идеальной жидкостью (рис. 1). Область газообразной среды представляет собой бесконечное полупространство, в то время как жидкость ограничена каналом прямоугольного поперечного сечения, имеющим абсолютно жесткие боковые и нижнюю стенки. Целью работы является исследование взаимного влияния скоростей жидкости $U$ и газа $U_{\infty}$ на границу аэро-/гидроупругой устойчивости при различных вариантах кинематических граничных условий, задаваемых на краях конструкции.

Малые деформации упругих пластин, возникающие в результате аэро-/ гидроупругого воздействия, определяются с помощью теории на основе ги-

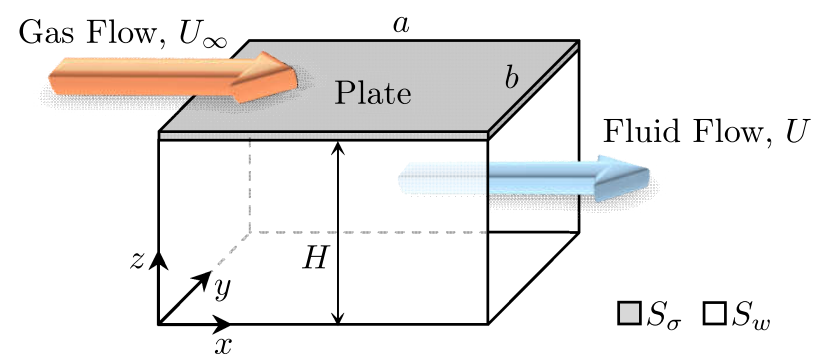

Рис. 1. Прямоугольная пластина, взаимодействующая с потоками газа и жидкости [Figure 1. Rectangular plate interacting with gas and fluid flows] 
потез Тимошенко [22]:

$$
\begin{aligned}
& \boldsymbol{\varepsilon}=\left\{\begin{array}{lllll}
\varepsilon_{x x} & \varepsilon_{y y} & \gamma_{x y} & \gamma_{x z} & \gamma_{y z}
\end{array}\right\}^{\top}=\tilde{\boldsymbol{\varepsilon}}+z \tilde{\boldsymbol{k}},
\end{aligned}
$$

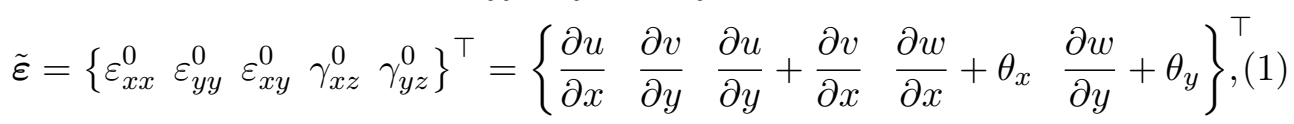

$$
\begin{aligned}
& \tilde{\boldsymbol{k}}=\left\{\begin{array}{lllll}
\varepsilon_{x x}^{1} & \varepsilon_{y y}^{1} & \varepsilon_{x y}^{1} & 0 & 0
\end{array}\right\}^{\top}=\left\{\begin{array}{lllll}
\frac{\partial \theta_{x}}{\partial x} & \frac{\partial \theta_{y}}{\partial y} & \frac{\partial \theta_{x}}{\partial y}+\frac{\partial \theta_{y}}{\partial x} & 0 & 0
\end{array}\right\}^{\top},
\end{aligned}
$$

где $u, v$ и $w$-перемещения точек серединной поверхности в направлении соответствующих осей системы координат $(x, y, z) ; \theta_{x}$ и $\theta_{y}$-углы поворота нормали вокруг осей $y$ и $x$.

Физические соотношения, устанавливающие связь между вектором усилий и моментов $\boldsymbol{T}$ и вектором деформаций $\boldsymbol{\varepsilon}$, представляются в матричном виде:

$$
\boldsymbol{T}=\left\{\begin{array}{llllllll}
N_{x x} & N_{y y} & N_{x y} & M_{x x} & M_{y y} & M_{x y} & Q_{x} & Q_{y}
\end{array}\right\}^{\top}=\mathbf{D} \boldsymbol{\varepsilon} .
$$

Элементы матрицы $\mathbf{D}$ для изотропного материала определяются через модуль упругости $E$, коэффициент Пуассона $\nu$ и модуль сдвига известным образом.

Математическая постановка задачи динамики пластины основана на вариационном принципе возможных перемещений, в который включаются слагаемые, учитывающие работу аэро-/ и гидродинамического давлений, а также сил инерции. В матричной форме он может быть записан следующим образом:

$$
\int_{S_{s}} \delta \varepsilon^{\top} \mathbf{D} \varepsilon d S+\int_{V_{s}} \rho_{s} \delta \boldsymbol{d}^{\top} \ddot{\boldsymbol{d}} d V-\int_{S_{\sigma}} \delta \boldsymbol{d}^{\top} \boldsymbol{P} d S,
$$

где $\rho_{s}$ - плотность материала пластины, $\boldsymbol{d}$ и $\boldsymbol{P}=\left\{\begin{array}{lllll}0 & 0 & p_{a}+p_{f} & 0 & 0\end{array}\right\}^{\top}-$ векторы обобщенных перемещений и поверхностных нагрузок; $p_{a}$ и $p_{f}$ - аэродинамическое и гидродинамическое давления, действующие на наружную и внутреннюю поверхности пластины; $S_{s}$ - поверхность, ограничивающая объем пластины $V_{s}$. Точками обозначены первая и вторая производная по времени.

Аэродинамическое давление, действующее со стороны потока газа на упругую поверхность $S_{s}$, вычисляется согласно квазистатической аэродинамической теории $[1,2]$ :

$$
\begin{gathered}
p_{a}=-\left(q \frac{\partial w}{\partial x}+q_{1} \frac{\partial w}{\partial t}\right) \\
q=\rho_{\infty} U_{\infty}^{2} / \beta, \quad q_{1}=q\left(\mathrm{M}_{\infty}^{2}-2\right) /\left(U_{\infty} \beta^{2}\right) .
\end{gathered}
$$

Здесь $\mathrm{M}_{\infty}=U_{\infty} / c_{\infty}$ - число Маха в газе; $\rho_{\infty}$ и $c_{\infty}-$ плотность и скорость звука в невозмущенном потоке газа; $q$ - модифицированное динамическое давление; $\beta=\left(\mathrm{M}_{\infty}^{2}-1\right)^{1 / 2}$.

Гидродинамическое давление, действующее со стороны идеальной жидкости на смоченную поверхность пластины $S_{\sigma}=S_{f} \cap S_{s}$, вычисляется с использованием линеаризованного уравнения Бернулли $[8,23]$ :

$$
p_{f}=-\rho_{f}\left(\frac{\partial \phi}{\partial t}+U \frac{\partial \phi}{\partial x}\right) .
$$


Здесь $\rho_{f}$ - плотность жидкости; $S_{f}$ - поверхность, ограничивающая объем жидкости $V_{f} ; \phi-$ потенциал возмущения скорости, который должен удовлетворять следующему уравнению:

$$
\nabla^{2} \phi=\frac{1}{c^{2}} \frac{\partial^{2} \phi}{\partial t^{2}}+\frac{2 U}{c^{2}} \frac{\partial^{2} \phi}{\partial t \partial x}+\mathrm{M}^{2} \frac{\partial^{2} \phi}{\partial x^{2}},
$$

где $c$ и М - скорость звука и число Маха в жидкости.

На границе жидкости с упругой пластиной задаются условия равенства нормальных скоростей и нормальных напряжений:

$$
\dot{w}=v_{n}, \quad \sigma_{n n}^{s}=\sigma_{n n}^{f} .
$$

Здесь $v_{n}$-скорость жидкости в направлении внешней нормали к плоскости $S_{\sigma}, \sigma_{n n}^{s}$ и $\sigma_{n n}^{f}-$ нормальные напряжения упругой конструкции и жидкости на границе контакта. В случае малых перемещений вектор нормали к плоскости $S_{\sigma}$ незначительно отклоняется от координаты $z$, тогда из граничных условий (7) получим

$$
\begin{gathered}
\left(\frac{\partial w}{\partial t}+U \frac{\partial w}{\partial x}\right)=\frac{\partial \phi}{\partial z} \\
\sigma_{n n}^{s}=p_{f} .
\end{gathered}
$$

На границе жидкости и жесткой стенки $S_{w}$ задается условие

$$
\partial \phi / \partial n=0
$$

Потенциал возмущения скорости на входе в канал и выходе из него подчиняется следующим граничным условиям:

$$
x=0: \phi=0, \quad x=a: \partial \phi / \partial x=0 .
$$

2. Численная реализация. Уравнение в частных производных для потенциала возмущения скорости (6) вместе с граничными условиями (9), (10) и условием непроницаемости (8) преобразуются с помощью слабой постановки метода Бубнова-Галеркина. Применение стандартных процедур МКЭ к полученному уравнению и выражению (3) с учетом (1), (2), (4), (5) приводит к следующему матричному соотношению:

$$
\mathbf{M}\left\{\begin{array}{ll}
\ddot{\boldsymbol{d}} & \ddot{\boldsymbol{\phi}}
\end{array}\right\}^{\top}+\mathbf{C}\left\{\begin{array}{ll}
\dot{\boldsymbol{d}} & \dot{\boldsymbol{\phi}}
\end{array}\right\}^{\top}+(\mathbf{K}+\mathbf{A})\left\{\begin{array}{ll}
\boldsymbol{d} & \boldsymbol{\phi}
\end{array}\right\}^{\top}=0,
$$

где

$$
\begin{array}{cc}
\mathbf{K}=\left[\begin{array}{cc}
\mathbf{K}_{s} & 0 \\
0 & \mathbf{K}_{f}
\end{array}\right], \quad \mathbf{M}=\left[\begin{array}{cc}
\mathbf{M}_{s} & 0 \\
0 & \mathbf{M}_{f}
\end{array}\right], \\
\mathbf{C}=\left[\begin{array}{cc}
\mathbf{C}_{s} & \mathbf{C}_{s f} \\
\mathbf{C}_{f s} & \mathbf{C}_{f}
\end{array}\right], \quad \mathbf{A}=\left[\begin{array}{cc}
\mathbf{A}_{s} & \mathbf{A}_{s f} \\
\mathbf{A}_{f s} & \mathbf{A}_{f}
\end{array}\right], \\
\mathbf{K}_{s}=\sum_{m_{s}} \int_{S_{s}} \mathbf{B}^{\top} \mathbf{D B} d S, & \mathbf{M}_{s}=\sum_{m_{s}} \int_{V_{s}} \rho_{s} \mathbf{N}^{\top} \mathbf{N} d V,
\end{array}
$$




$$
\begin{gathered}
\mathbf{M}_{f}=\sum_{m_{f}} \int_{V_{f}} \frac{\mathbf{F}^{\top} \mathbf{F}}{c^{2}} d V, \quad \mathbf{K}_{f}=\sum_{m_{f}} \int_{V_{f}}\left(\mathbf{F}^{\top},_{x} \mathbf{F},,_{x}+\mathbf{F}_{, y}^{\top} \mathbf{F},{ }_{y}+\mathbf{F}_{, z}^{\top} \mathbf{F}, z\right) d V, \\
\mathbf{C}_{f s}=-\sum_{m_{s}} \int_{S_{\sigma}} \mathbf{F}^{\top} \overline{\mathbf{N}} d S, \quad \mathbf{C}_{s f}=\sum_{m_{s}} \int_{S_{\sigma}} \rho_{f} \overline{\mathbf{N}}^{\top} \mathbf{F} d S, \\
\mathbf{A}_{s f}=\sum_{m_{s}} \int_{S_{\sigma}} \rho_{f} U \overline{\mathbf{N}}^{\top} \mathbf{F},{ }_{x} d S, \quad \mathbf{A}_{f}=-\sum_{m_{f}} \int_{V_{f}} \mathrm{M}^{2} \mathbf{F}^{\top}{ }_{x} \mathbf{F},{ }_{x} d V, \\
\mathbf{C}_{f}=\sum_{m_{f}} \int_{V_{f}} \frac{2 U}{c^{2}} \mathbf{F}^{\top},{ }_{x} \mathbf{F} d V, \quad \mathbf{C}_{s}=\sum_{m_{s}} \int_{S_{s}} q_{1} \overline{\mathbf{N}}^{\top} \overline{\mathbf{N}} d S, \\
\mathbf{A}_{f s}=-\sum_{m_{s}} \int_{S_{\sigma}} U \mathbf{F}^{\top} \overline{\mathbf{N}},{ }_{x} d S, \quad \mathbf{A}_{s}=\sum_{m_{s}} \int_{S_{s}} q \overline{\mathbf{N}}^{\top} \overline{\mathbf{N}},{ }_{x} d S .
\end{gathered}
$$

Здесь $m_{f}$ и $m_{s}$ - число конечных элементов, на которые разбиваются области жидкости и пластины, $\mathbf{N}, \mathbf{F}$ и $\overline{\mathbf{N}}$ - матрицы функций формы для конечного элемента пластины, потенциала возмущения скорости и нормальной составляющей вектора перемещений пластины.

Рассмотрим возмущенное движение конструкции и жидкости в виде

$$
(\boldsymbol{d}, \boldsymbol{\phi})=(\boldsymbol{q}, \boldsymbol{f}) \exp (\lambda t)
$$

где $\boldsymbol{q}$ и $\boldsymbol{f}$ - некоторые функции координат, $\lambda=\delta+i \omega-$ характеристический показатель. Здесь полагается, что $\omega$ - собственная частота колебаний, а $\delta$ характеризует демпфирование. В результате исходная система уравнений (11) преобразуется к обобщенной задаче на собственные значения вида

$$
\left[\begin{array}{cc}
\mathbf{C} & \mathbf{K}+\mathbf{A} \\
-\mathbf{I} & 0
\end{array}\right]\left\{\begin{array}{c}
\lambda \boldsymbol{x} \\
\boldsymbol{x}
\end{array}\right\}+\lambda\left[\begin{array}{cc}
\mathbf{M} & 0 \\
0 & \mathbf{I}
\end{array}\right]\left\{\begin{array}{c}
\lambda \boldsymbol{x} \\
\boldsymbol{x}
\end{array}\right\}=0
$$

где $\mathbf{I}$ - единичная матрица, $\boldsymbol{x}=\left\{\begin{array}{ll}\boldsymbol{q} & \boldsymbol{f}\end{array}\right\}^{\top}$.

Вычисление комплексных собственных значений связанной системы двух уравнений (12) осуществляется с помощью алгоритма на основе неявно перезапускаемого метода Арнольди [24]. Оценка устойчивости основана на анализе комплексных собственных значений задачи (12), полученной при последовательно возрастающей скорости течения жидкости или газа.

3. Результаты расчетов. В численных примерах рассматривается выполненная из алюминия прямоугольная пластина со следующими вариантами кинематических граничных условий (ГУ): свободный край $(\mathrm{F})$ и жесткое закрепление $\left(u=v=w=\theta_{x}=\theta_{y}=0, \mathrm{C}\right)$. При указании заданной комбинации ограничений перечисление ведется по часовой стрелке начиная с левого края. Например, вариант CFFF соответствует консольной пластине, закрепленной только на стороне, где втекает жидкость. В расчетах варьируемыми переменными выступают модифицированное динамическое давление газа $q$ $\left(\rho_{\infty}=0.413 \mathrm{\kappa г} / \mathrm{m}^{3}, c_{\infty}=299.5 \mathrm{~m} / \mathrm{c}, \mathrm{M}_{\infty}=3\right)$ и скорость течения жидкости $U\left(\rho_{f}=1000 \mathrm{kг} / \mathrm{m}^{3}, c=1500 \mathrm{~m} / \mathrm{c}\right)$. Все остальные геометрические и физикомеханические параметры модели аналогичны таковым в работах $[17,18]$. Для 
отображения полученных результатов используются следующие безразмерные величины (собственное значение $\Lambda$, скорость $\Upsilon$ и динамическое давление $\gamma)$ :

$$
\begin{gathered}
\Lambda=\Lambda_{\mathrm{Re}}+i \Lambda_{\mathrm{Im}}=a^{2} \sqrt{\frac{\rho_{s} h}{D}} \lambda, \\
\Upsilon=a \sqrt{\frac{\rho_{s} h}{D}} U, \\
\gamma=q \frac{a^{3}}{D} \times 10^{-3}, \quad D=\frac{E h^{3}}{12\left(1-\nu^{2}\right)} .
\end{gathered}
$$

Достоверность решения задачи аэроупругой устойчивости подтверждена сравнением с известными численными [15,25] и аналитическим [1] результатами. Полученные значения динамического давления $\gamma$ и соответствующие им частоты флаттера $\Lambda$ приведены в таблице. Здесь под SSSS обозначен следующий вариант закрепления прямоугольной $(a / b=1)$ пластины:

$$
\begin{array}{ll}
x=0, a: & v=w=\theta_{x}=0 ; \\
y=0, b: & u=w=\theta_{y}=0 .
\end{array}
$$

Из представленных данных следует, что полученные в рамках разработанного конечно-элементного алгоритма результаты хорошо согласуются с ранее опубликованными данными. В ходе сравнения полагалось, что второе слагаемое в выражении (4) равно нулю (аэродинамическое демпфирование отсутствует).

Верификация алгоритма для задач о собственных колебаниях и гидроупругой устойчивости одной или нескольких параллельных пластин, взаимодействующих с жидкостью, представлена в предыдущих работах авторов $[17,18]$.

Исследование взаимного влияния скоростей течения жидкости и газа на границу аэро-/гидроупругой устойчивости пластины основано на построении диаграмм в области параметров $\Upsilon-\gamma$ (см. рис. 2). Такое представление результатов позволяет получить полную картину поведения системы, но требует значительных вычислительных затрат. В связи с этим было рассмотрено только два варианта граничных условий (СССС и ССFC), способных отразить основные особенности. При указанных способах закрепления и отсутствии внешнего потока газа потеря устойчивости осуществляется в виде

Сравнение критических давлений $\gamma_{\text {cr }}$ и частот флаттера $\Lambda_{c r}$ [Comparison of critical dynamic pressure $\gamma_{\mathrm{cr}}$ and flutter frequencies $\Lambda_{\mathrm{cr}}$ ]

\begin{tabular}{c|c|c|c|c|c}
\hline $\begin{array}{c}\text { Boundary } \\
\text { conditions }\end{array}$ & Parameters & $\begin{array}{c}\text { Present } \\
\text { paper }\end{array}$ & FEM [25] & FEM [15] & $\begin{array}{c}\text { Analytical } \\
\text { solution [1] }\end{array}$ \\
\hline \multirow{2}{*}{$\mathrm{CCCC}$} & $\gamma_{\mathrm{cr}} \times 10^{3}$ & 849.1 & 850.418 & 852.34 & - \\
\hline \multirow{2}{*}{$\mathrm{SSSS}$} & $\Lambda_{\mathrm{cr}}^{2} \times 10^{3}$ & 4280.2 & 4282.03 & 4274.32 & - \\
\hline$\Lambda_{\mathrm{cr}}^{2}$ & 1846.7 & 511.786 & 511.11 & 512.65 \\
& \multicolumn{1}{c}{1843.29} & 1840.29 & 1848.21 \\
\hline
\end{tabular}



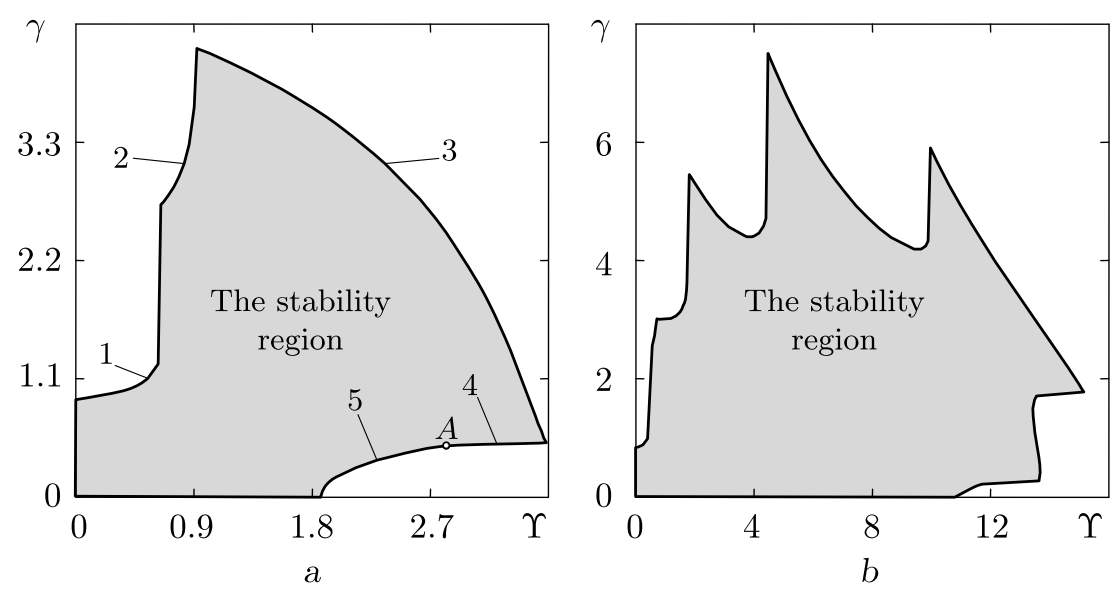

Рис. 2. Диаграммы устойчивости пластины, расположенной на слое текущей жидкости и обтекаемой сверхзвуковым потоком газа, полученные при различных вариантах граничных условий: $a-\mathrm{CCCC} ; b-\mathrm{CCFC}$ [Figure 2. Stability diagrams for the plate placed on the layer of flowing fluid and subjected to the external supersonic gas flow for different types of boundary conditions: a - the CCCC boundary conditions; $b$ - the CCFC boundary conditions]

дивергенции $(\Upsilon=1.875)$ и одномодового флаттера $(\Upsilon=10.782)$ соответственно. Учет аэродинамической нагрузки приводит к стабилизации системы, даже если скорость течения жидкости $\Upsilon$ больше критической. Представленные на рис. 2 диаграммы устойчивости демонстрируют это явление. Однако чрезмерно большая величина параметра $\gamma$ при фиксированном значении $\Upsilon$ приводит к возникновению неустойчивости. Таким образом, система остается устойчивой, только если точка с координатами $(\Upsilon, \gamma)$ попадает внутрь закрашенной области.

Характерной особенностью обеих диаграмм, приведенных на рис. 2, является наличие изломов и вогнутых участков. Причина их появления обусловлена или сменой моды, по которой происходит потеря устойчивости, или сменой типа неустойчивости. На рис. 2, а участки границы, которым ставятся в соответствие разные моды, обозначены цифрами 1-3, а на рис. 3 приведены соответствующие им формы колебаний. В зависимости от параметров течения обоих потоков мода, по которой осуществляется потеря устойчивости, меняется, формируя таким образом границу с резкими изломами. Другая причина нарушения гладкости имеет место на участке 5-4-3. Здесь дивергенция сменяется одномодовым флаттером в точке $A$. Для граничных условий CCFC характерна аналогичная картина, но с более выраженными пиками (см. рис. $2, b)$. При этом варианте закрепления потеря устойчивости всегда осуществлялась в виде флаттера по одной моде.

Еще одним фактором, заметно влияющим на границу устойчивости рассматриваемых конструкций, является высота слоя жидкости $H$. В случае отсутствия внешнего потока газа для граничных условий вида СССС и ССFС асимптотическое поведение критических скоростей $\Upsilon$ от отношения $H / b$ наступает при $H / b>0.5$ и $H / b>0.25$ соответственно (см. рис. 4 , пунктирные линии). На рис. 4 сплошной линией обозначены аналогичные зависимости, полученные при фиксированной величине аэродинамического давления $\gamma$. Из представленных данных видно различие в результатах, полученных с уче- 


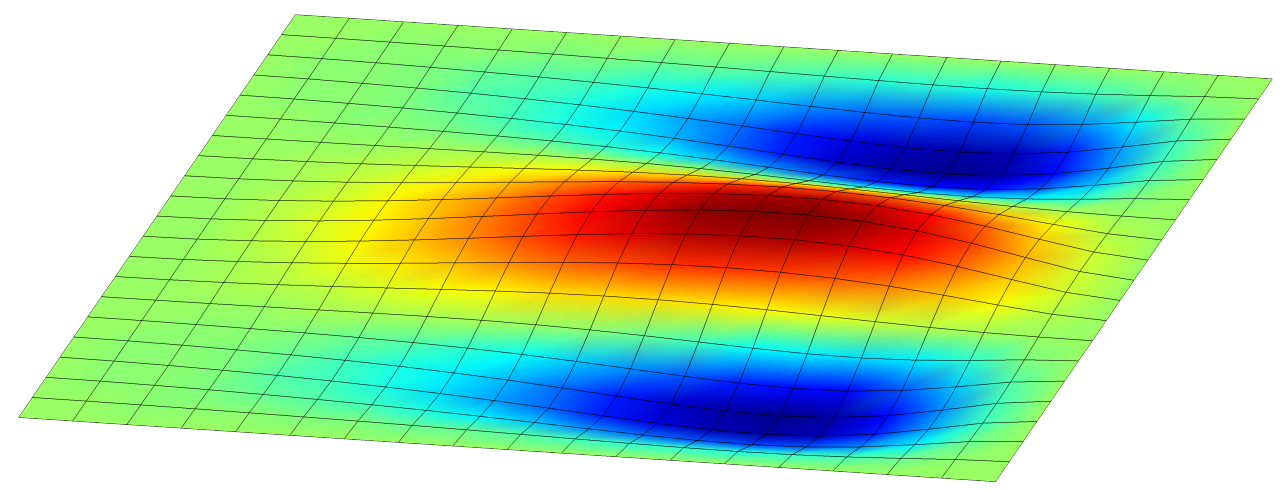

a

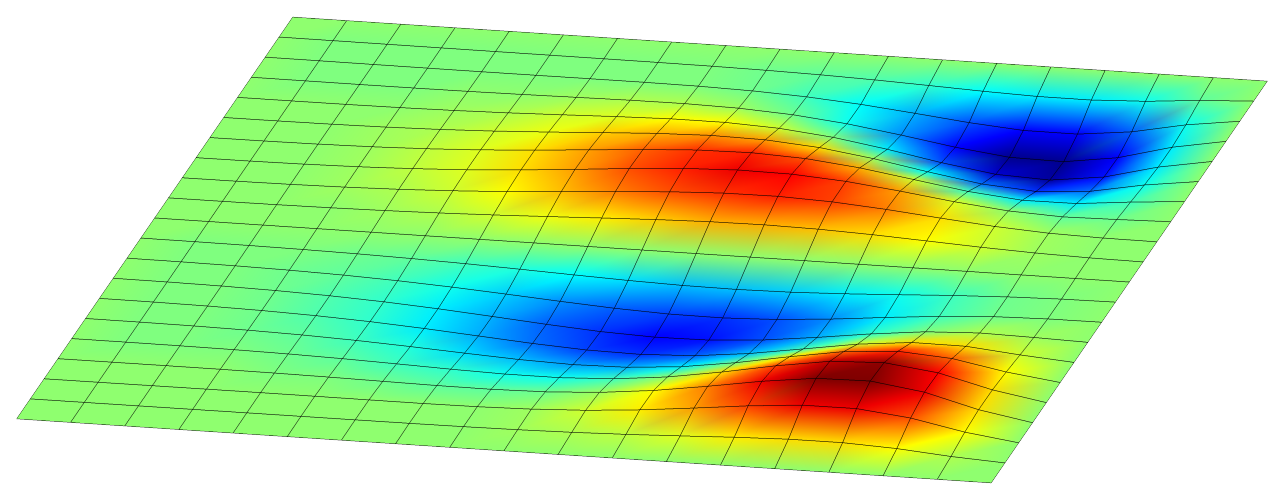

$b$

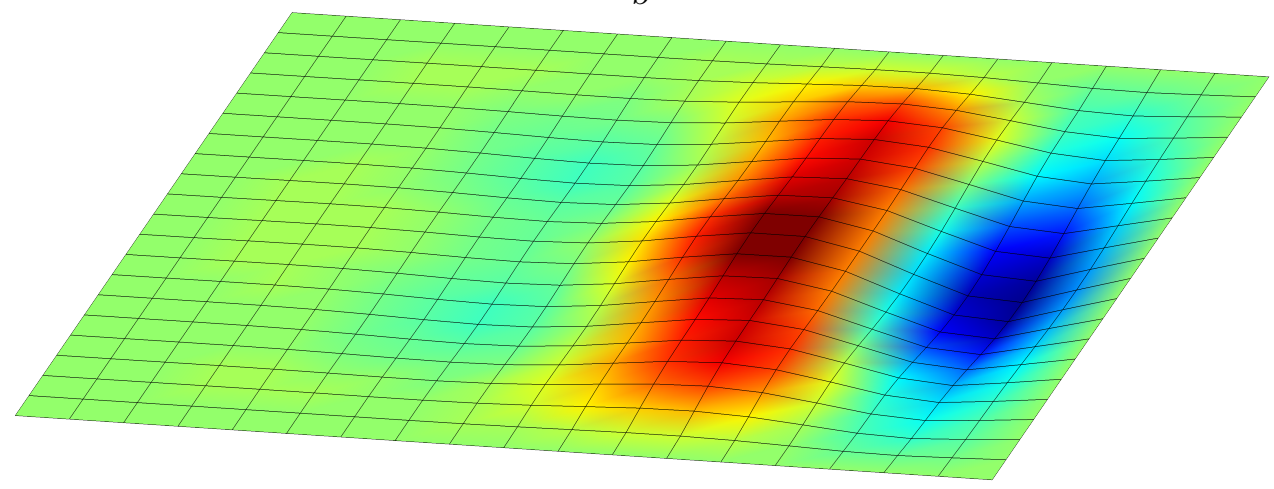

$c$

Рис. 3. Формы колебаний, по которым осуществляется потеря устойчивости: $\mathrm{a}-\mathrm{yчасток} 1$; b-участок 2; c-участок 3 (см. рис. 2, a) [Figure 3. Mode shapes on which the stability is loss: $a$-the segment $1 ; b$-the segment $2 ; c$-the segment 3 (see Fig. 2, a)] 

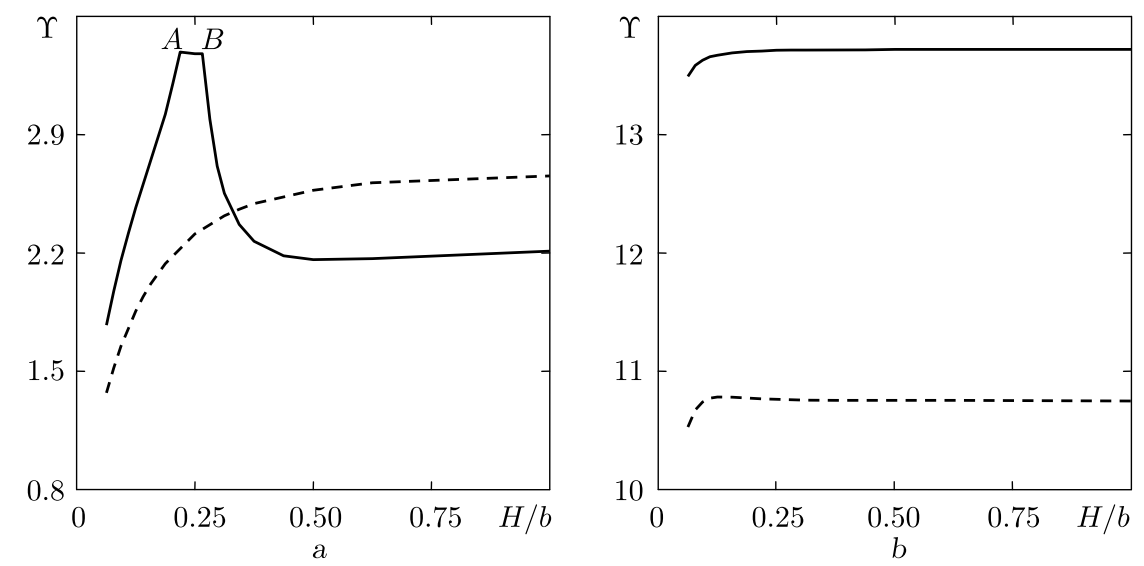

Рис. 4. Зависимости безразмерных критических скоростей $\Upsilon$ от высоты слоя жидкости $H$ : $a-\mathrm{CCCC} ; b-\mathrm{CCFC}$. Сплошные линии при $\gamma=0.4$, пунктирные линии при $\gamma=0$

[Figure 4. Dependencies of dimensionless critical velocities $\Upsilon$ of the fluid height $H$ : a-the CCCC boundary conditions; $b$ - the CCFC boundary conditions. Solid lines when $\gamma=0.4$, dashed lines when $\gamma=0$ ]

том и без учета внешнего обтекания. Для жестко закрепленной по всем сторонам пластины (см. рис. 4, а) немонотонное поведение кривой обусловлено сменой типа потери устойчивости с дивергенции на одномодовый флаттер в точке $A$ и изменением моды в точке $B$. Кроме этого, начиная с $H / b>0.32$ стабилизирующее влияние потока газа сменяется на дестабилизирующее. При другом варианте граничных условий (см. рис. 4, b) существенное повышение критических скоростей $\Upsilon$ имеет место на всем интервале изменения отношения $H / b$. Смены типа потери устойчивости в этом случае не происходит - на всем диапазоне рассмотренных значений имеет место флаттер по одной моде.

Заключение. Представлены результаты численного исследования динамической устойчивости прямоугольной пластины, взаимодействующей сверху со сверхзвуковым потоком газа и снизу с текущей идеальной жидкостью. Проанализировано влияние кинематических граничных условий, задаваемых на краях пластины, высоты слоя жидкости и взаимного влияния скоростей обоих потоков на границу аэро-/гидроупругой устойчивости. Из анализа полученных диаграмм устойчивости установлено, что внешний сверхзвуковой поток воздуха оказывает стабилизирующее воздействие и повышает границу гидроупругой устойчивости. Данный эффект имеет место в ограниченном диапазоне динамического давления газа и зависит от отношения скоростей обоих потоков. Установлено, что тип потери устойчивости определяется граничными условиями для пластины, высотой слоя жидкости и параметрами течения.

Декларация о финансовых и других взаимоотношениях. Работа выполнена при поддержке Российского фонда фундаментальных исследований (проект № 15-01-05254-а), гранта Президента Российской Федерации для государственной поддержки молодых российских ученых - кандидатов наук (МК-6167.2015.1) и программы УрО РАН (проект № 1510-1-18). Все авторы принимали участие в разработке концепции статьи и в написании рукописи. Авторы несут полную ответственность за предоставление окончательной рукописи в печать. Окончательная версия рукописи была одобрена всеми авторами. Авторы не получали гонорар за статью. 


\section{ORCIDs}

Сергей Аркадьевич Бочкарев: http://orcid.org/0000-0002-9722-1269

Сергей Владимирович Лекомцев: http://orcid.org/0000-0002-8331-2979

\section{БИБЛИОГРАФИЧЕСКИЙ СПИСОК}

1. Dowell E. H. Aeroelasticity of Plates and Shells / Mechanics: Dynamical Systems. vol. 1. Netherlands: Springer, 1975. xiii +139 pp.

2. Ильюшин А. А. Закон плоских сечений в аэродинамике больших сверхзвуковых скоростей // ПММ, 1956. Т. 20, №6. С. 733-755.

3. Новичков Ю. Н. Флаттер пластин и оболочек / Итоги науки и техники / Механика деформируемого твердого тела, Т. 11. М.: ВИНИТИ, 1978. С. 67-122.

4. Dowell E. H., Voss H. M. Theoretical and experimental panel flutter studies in the Mach number range 1.0 to $5.0 / / A I A A J ., 1965$. vol. 3, no. 12. pp. 2292-2304. doi: 10.2514/3. 3359.

5. Dixon S. C. Comparison of panel flutter results from approximate aerodynamic theory with results from exact inviscid theory and experiment: NASA TN D-3649; Technical Report no. 19660029122, 1966, https://ntrs.nasa.gov/search.jsp?R=19660029122.

6. Веденеев В. В., Гувернюк С. В., Зубков А. Ф., Колотников М. Е. Экспериментальное исследование одномодового панельного флаттера в сверхзвуковом потоке газа // Изв. РАН. МЖГ, 2010. № 2. С. 161-175.

7. Bismarck-Nasr M. N. Finite element analysis of aeroelasticity of plates and shells // Appl. Mech. Rev., 1992. vol. 45, no. 12. pp. 461-482. doi: 10.1115/1.3119783.

8. Païdoussis M. P. Fluid-structure Interactions: Slender Structures and Axial Flow, 2nd edition. vol. 2. London: Academic Press, 2014. 923 pp. doi: 0.1016/B978-0-12-397333-7. 09967-8.

9. Kornecki A., Dowell E. H., O'Brien J. On the aeroelastic instability of two-dimensional panels in uniform incompressible flow // Journal of Sound and Vibration, 1976. vol. 47, no. 2. pp. 163-178. doi: 10.1016/0022-460X (76)90715-X.

10. Dowell E. H. Nonlinear oscillations of a fluttering plate// AIAA J., 1966. vol. 4, no. 7. pp. 1267-1275. doi: 10.2514/3.3658.

11. Dowell E. H. Nonlinear oscillations of a fluttering plate. II // AIAA J., 1967. vol. 5, no. 10. pp. 1856-1862. doi: 10.2514/3.4316.

12. Mei C. A finite-element approach for nonlinear panel flutter // AIAA J., 1977. vol. 15, no. 8. pp. 1107-1110. doi: 10.2514/3.60760.

13. Yang T. Y., Han A. D. Nonlinear panel flutter using high-order triangular finite elements // AIAA J., 1983. vol.21, no. 10. pp. 1453-1461. doi: 10.2514/3.8267.

14. Haddadpour H., Navazi H. M., Shadmehri F. Nonlinear oscillations of a fluttering functionally graded plate// Compos. Struct., 2007. vol.79, no. 2. pp. 242-250. doi: 10.1016/j . compstruct.2006.01.006.

15. Prakash T., Ganapathi M. Supersonic flutter characteristics of functionally graded flat panels including thermal effects // Compos. Struct., 2006. vol.72, no.1. pp. 10-18. doi: 10. 1016/j . compstruct. 2004.10.007.

16. Rossettos J. N., Tong P. Finite-element analysis of vibration and flutter of cantilever anisotropic plates // J. Appl. Mech., 1974. vol.41, no.4. pp. 1075-1080. doi: 10.1115/1. 3423437.

17. Бочкарев С. А., Лекомцев С. В., Матвеенко В. П. Гидроупругая устойчивость прямоугольной пластины, взаимодействующей со слоем текущей идеальной жидкости // Изв. РАН. МЖГ, 2016. №6. C. 108-120. doi : 10.7868/S0568528116060049.

18. Бочкарев С. А., Лекомцев С. В. Численное исследование влияния граничных условий на гидроупругую устойчивость двух параллельных пластин, взаимодействующих со слоем текущей идеальной жидкости // Вычислительная механика сплошных сред, 2015. T. 8, № 4. C. 423-432. doi : 10.7242/1999-6691/2015.8.4.36. 
19. Kerboua Y., Lakis A. A., Thomas M., Marcouiller L. Modeling of plates subjected to a flowing fluid under various boundary conditions // Eng. Appl. Comp. Fluid Mech., 2008. vol. 2, no. 4. pp. 525-539. doi: 10.1080/19942060.2008.11015249.

20. Noorian M., Haddadpour H., Firouz-Abadi R. Investigation of panel flutter under the effect of liquid sloshing // J. Aerosp. Eng., 2015. vol. 28, no. 2, 04014059. doi : 10.1061/(ASCE) AS. 1943-5525.0000384.

21. Бочкарев С. А., Лекомцев С. В. Аэроупругая устойчивость круговых цилиндрических оболочек, содержащих текущую жидкость // Вестн. Сам. гос. техн. ун-та. Сер. Физ.мат. науки, 2015. Т. 19, № 4. С. 750-767. doi: 10.14498/vsgtu1453.

22. Вольмир А. С. Нелинейная динамика пластин и оболочек. М.: Наука, 1972. 432 с.

23. Вольмир А. С. Оболочки в потоке жидкости и газа. Задачи гидроупругости. М.: Наука, 1979. 320 c.

24. Lehoucq R. B., Sorensen D. C. Deflation techniques for an implicitly restarted Arnoldi iteration// SIAM J. Matrix Anal. Appl., 1996. vol.17, no.4. pp. 789-821. doi:10.1137/ S0895479895281484.

25. Olson M. D. Some flutter solutions using finite elements // AIAA J., 1970. vol.8, no. 4. pp. 747-752. doi: 10.2514/3.5751.

Поступила в редакцию 13/VII/2016; в окончательном варианте - 23/VIII/2016; принята в печать - 09/IX/2016. 


\title{
MSC: 74F10
}

\section{AEROELASTIC STABILITY OF PLATE INTERACTING WITH A FLOWING FLUID}

\section{S. A. Bochkarev, S. V. Lekomtsev}

Institute of Continuous Media Mechanics, Ural Branch of RAS, 1, Akad. Korolyova st., Perm, 614013, Russian Federation.

\begin{abstract}
The paper presents the results of a numerical study of the dynamic behavior of the deformable plate interacting both with the external supersonic gas flow and the internal fluid flow. The constitutive relations describing the behavior of ideal compressible fluid in the case of small perturbations are written in terms of the perturbation velocity potential and transformed using the Bubnov-Galerkin method. The aero- and dynamic pressures are calculated based on the quasi-static aerodynamic theory. The strains in the plate evaluated following the Timoshenko hypotheses. A mathematical formulation of the dynamic problem of elastic structure is developed using the variational principle of virtual displacements, which takes into account the work done by the inertia forces, aerodynamic and hydrodynamic pressures. Calculation of complex eigenvalues of the coupled system of two equations is performed using an algorithm based on implicitly restarted Arnoldi method. The stability criterion is based on an analysis of the complex eigenvalues of system of two equations obtained for increasing flow or gas velocity. The reliability of the obtained numerical solution has been estimated by comparing it with the available theoretical data. A few numerical examples were considered to demonstrate the existence of different types of instability depending on the velocities of fluid or gas flow, combinations of kinematic boundary conditions prescribed at the edges of the plate, and the fluid layer height. It has been found that a violation of the smoothness of the obtained relationships and diagrams of stability is caused by a change in the flutter mode, or change of the type of loss of stability.
\end{abstract}

Keywords: aeroelasticity, supersonic gas flow, potential flow of compressible fluid, rectangular plate, finite-element method, stability, flutter.

(C) 2016 Samara State Technical University.

\section{Please cite this article in press as:}

B ochkarev S. A., Lekomtsev S. V. Aeroelastic stability of plate interacting with a flowing fluid, Vestn. Samar. Gos. Tekhn. Univ., Ser. Fiz.-Mat. Nauki [J. Samara State Tech. Univ., Ser. Phys. \& Math. Sci.], 2016, vol. 20, no. 3, pp. 552-566. doi: 10.14498/vsgtu1510. (In Russian)

\section{Authors Details:}

Sergey A. Bochkarev (Cand. Phys. \& Math. Sci.; bochkarev@icmm.ru; Corresponding Author), Senior Researcher, Dept. of Complex Problems of Mechanics of Deformable Bodies.

Sergey V. Lekomtsev (Cand. Phys. \& Math. Sci.; lekomtsev@icmm.ru), Researcher, Dept. of Complex Problems of Mechanics of Deformable Bodies. 
Declaration of Financial and Other Relationships. This work was supported by the Russian Foundation for Basic Research (project no. 15-01-05254-a), by the grant of the President of Russian Federation for state support of young Russian scientists (project no. MK-6167.2015.1), and by the Ural Branch of RAS program (project no. 15-10-1-18). Each author has participated in the article concept development and in the manuscript writing. The authors are absolutely responsible for submitting the final manuscript in print. Each author has approved the final version of manuscript. The authors have not received any fee for the article.

\section{ORCIDs}

Sergey A. Bochkarev: http://orcid.org/0000-0002-9722-1269

Sergey V. Lekomtsev: http://orcid.org/0000-0002-8331-2979

\section{REFERENCES}

1. Dowell E. H. Aeroelasticity of Plates and Shells, Mechanics: Dynamical Systems, vol.1. Netherlands, Springer, 1975, xiii +139 pp.

2. Ilyushin A. A. The law of plane sections in aerodynamics of the high supersonic velocities, Prikl. Mat. Mekh, 1956, vol. 20, no. 6, pp. 733-755 (In Russian).

3. Novichkov Yu. N. Flutter of plates and shells, Itogi nauki i tekhniki [Advances in Science and Technology], Mechanics of Deformable Solids, 11. Moscow, VINITI, 1978, pp. 67-122 (In Russian).

4. Dowell E. H., Voss H. M. Theoretical and experimental panel flutter studies in the Mach number range 1.0 to 5.0, AIAA J., 1965, vol. 3, no. 12, pp. 2292-2304. doi : 10.2514/3. 3359.

5. Dixon S. C. Comparison of panel flutter results from approximate aerodynamic theory with results from exact inviscid theory and experiment, NASA TN D-3649; Technical Report no. 19660029122, 1966, https://ntrs.nasa.gov/search.jsp?R=19660029122.

6. Vedeneev V. V., Guvernyuk S. V., Zubkov A. F., Kolotnikov M. E. Experimental investigation of single-mode panel flutter in supersonic gas flow, Fluid Dyn., 2010, vol. 45, no. 2, pp. 312-324. doi : 10.1134/S001546281002016X.

7. Bismarck-Nasr M. N. Finite element analysis of aeroelasticity of plates and shells, Appl. Mech. Rev., 1992, vol.45, no. 12, pp. 461-482. doi: 10.1115/1.3119783.

8. Païdoussis M. P. Fluid-structure Interactions: Slender Structures and Axial Flow, 2nd edition, vol. 2. London, Academic Press, 2014, 923 pp. doi: 0.1016/B978-0-12-397333-7. 09967-8.

9. Kornecki A., Dowell E. H., O'Brien J. On the aeroelastic instability of two-dimensional panels in uniform incompressible flow, Journal of Sound and Vibration, 1976, vol. 47, no. 2, pp. 163-178. doi: 10.1016/0022-460X (76)90715-X.

10. Dowell E. H. Nonlinear oscillations of a fluttering plate, AIAA J., 1966, vol.4, no.7, pp. 1267-1275. doi : 10.2514/3.3658.

11. Dowell E. H. Nonlinear oscillations of a fluttering plate. II, AIAA J., 1967, vol. 5, no. 10, pp. 1856-1862. doi: 10.2514/3.4316.

12. Mei C. A finite-element approach for nonlinear panel flutter, AIAA J., 1977, vol.15, no.8, pp. 1107-1110. doi : 10.2514/3.60760.

13. Yang T. Y., Han A. D. Nonlinear panel flutter using high-order triangular finite elements, AIAA J., 1983, vol. 21, no. 10, pp. 1453-1461. doi: 10.2514/3.8267.

14. Haddadpour H., Navazi H. M., Shadmehri F. Nonlinear oscillations of a fluttering functionally graded plate, Compos. Struct., 2007, vol.79, no.2, pp. 242-250. doi: 10.1016/j . compstruct.2006.01.006.

15. Prakash T., Ganapathi M. Supersonic flutter characteristics of functionally graded flat panels including thermal effects, Compos. Struct., 2006, vol.72, no. 1, pp. 10-18. doi: 10.1016/ j.compstruct.2004.10.007.

16. Rossettos J. N., Tong P. Finite-element analysis of vibration and flutter of cantilever anisotropic plates, J. Appl. Mech., 1974, vol.41, no.4, pp. 1075-1080. doi: 10.1115/1. 3423437. 
17. Bochkarev S. A., Lekomtsev S. V., Matveenko V. P. Hydroelastic stability of rectangular plate interacting with a layer of ideal flowing fluid, Fluid Dyn., 2016, vol.51, no. 6 (to appear).

18. Bochkarev S. A., Lekomtsev S. V. Numerical investigation of the effect of boundary conditions on hydroelastic stability of two parallel plates interacting with a layer of ideal flowing fluid, Comput. Continuum Mech., 2015, vol.8, no.4, pp. 423-432 (In Russian). doi: 10.7242/1999-6691/2015.8.4.36.

19. Kerboua Y., Lakis A. A., Thomas M., Marcouiller L. Modeling of plates subjected to a flowing fluid under various boundary conditions, Eng. Appl. Comp. Fluid Mech., 2008, vol. 2, no. 4, pp. 525-539. doi: 10.1080/19942060.2008.11015249.

20. Noorian M., Haddadpour H., Firouz-Abadi R. Investigation of panel flutter under the effect of liquid sloshing, J. Aerosp. Eng., 2015, vol. 28, no. 2, 04014059. doi: 10.1061/(ASCE) AS. 1943-5525.0000384.

21. Bochkarev S. A., Lekomtsev S. V. An aeroelastic stability of the circular cylindrical shells containing a flowing fluid, Vestn. Samar. Gos. Tekhn. Univ., Ser. Fiz.-Mat. Nauki [J. Samara State Tech. Univ., Ser. Phys. \& Math. Sci.], 2015, vol. 19, no. 4, pp. 750-767 (In Russian). doi: 10.14498/vsgtu1453.

22. Vol'mir A. S. Nelineinaia dinamika plastin i obolochek [Nonlinear Dynamics of Plates and Shells]. Moscow, Nauka, 1972, 432 pp. (In Russian)

23. Vol'mir A. S. Obolochki v potoke zhidkosti i gaza. Zadachi gidrouprugosti [Shells in Fluid and Gas Flow. Problems of Hydroelasticity]. Moscow, Nauka, 1979, 320 pp. (In Russian)

24. Lehoucq R. B., Sorensen D. C. Deflation techniques for an implicitly restarted Arnoldi iteration, SIAM J. Matrix Anal. Appl., 1996, vol.17, no.4, pp. 789-821. doi: 10.1137/ S0895479895281484.

25. Olson M. D. Some flutter solutions using finite elements, AIAA J., 1970, vol.8, no. 4, pp. 747-752. doi: 10.2514/3.5751.

Received 13/VII/2016;

received in revised form $23 / \mathrm{VIII} / 2016$;

accepted 09/IX/2016. 